PREPARED FOR THE U.S. DEPARTMENT OF ENERGY, UNDER CONTRACT DE-AC02-76CH03073

PPPL-3712

PPPL-3712

UC-70

Lagrangian Description of Nonadiabatic Particle Motion in Spherical Tori

by

Yu.V. Yakovendo, Ya.I. Kolesnichenko, and R.B. White

June 2002

N/M

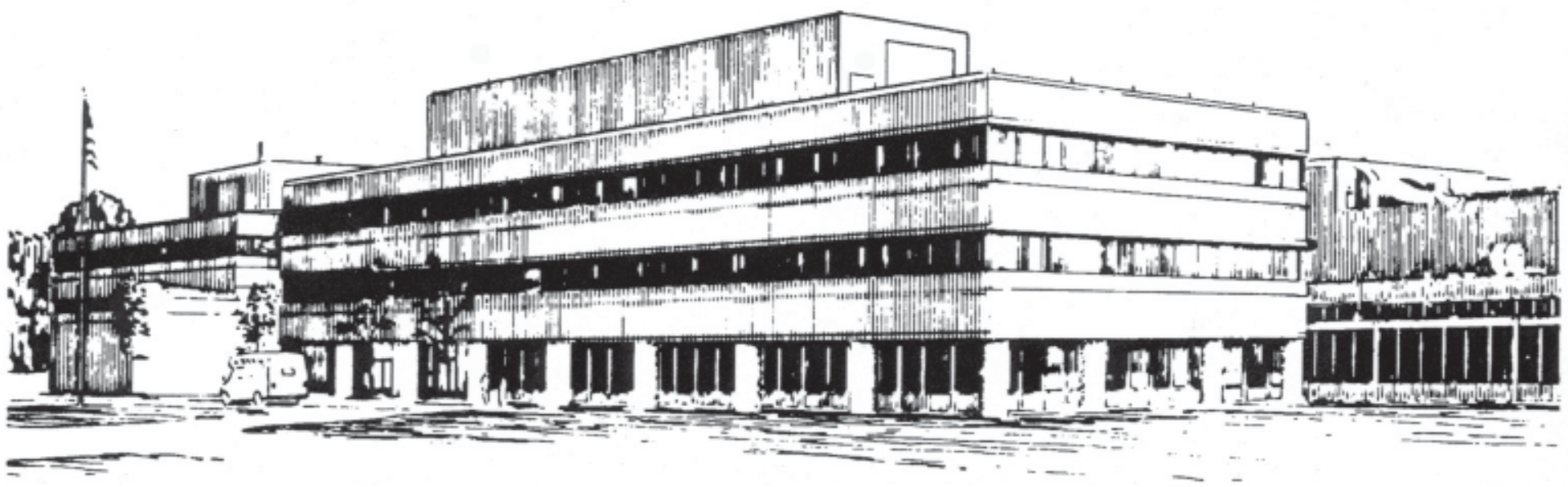

PRINCETON PLASMA PHYSICS LABORATORY PRINCETON UNIVERSITY, PRINCETON, NEW JERSEY 


\section{PPPL Reports Disclaimer}

This report was prepared as an account of work sponsored by an agency of the United States Government. Neither the United States Government nor any agency thereof, nor any of their employees, makes any warranty, express or implied, or assumes any legal liability or responsibility for the accuracy, completeness, or usefulness of any information, apparatus, product, or process disclosed, or represents that its use would not infringe privately owned rights. Reference herein to any specific commercial product, process, or service by trade name, trademark, manufacturer, or otherwise, does not necessarily constitute or imply its endorsement, recommendation, or favoring by the United States Government or any agency thereof. The views and opinions of authors expressed herein do not necessarily state or reflect those of the United States Government or any agency thereof.

\section{Availability}

This report is posted on the U.S. Department of Energy's Princeton Plasma Physics Laboratory Publications and Reports web site in Fiscal Year 2002. The home page for PPPL Reports and Publications is: http://www.pppl.gov/pub_report/

DOE and DOE Contractors can obtain copies of this report from:

U.S. Department of Energy

Office of Scientific and Technical Information

DOE Technical Information Services (DTIS)

P.O. Box 62

Oak Ridge, TN 37831

Telephone: (865) 576-8401

Fax: (865) 576-5728

Email: reports@adonis.osti.gov

This report is available to the general public from:

National Technical Information Service

U.S. Department of Commerce

5285 Port Royal Road

Springfield, VA 22161

Telephone: 1-800-553-6847 or

(703) 605-6000

Fax: (703) 321-8547

Internet: http://www.ntis.gov/ordering.htm 


\title{
Lagrangian Description of Nonadiabatic Particle Motion in Spherical Tori
}

\author{
$\underline{\text { Yu. V. Yakovenko }}^{1}$, Ya. I. Kolesnichenko ${ }^{1}$, and R. B. White ${ }^{2}$ \\ ${ }^{1}$ Institute for Nuclear Research, Prospekt Nauky 47, Kyiv 03680, Ukraine \\ 2 Princeton Plasma Physics Laboratory, P.O. Box 451, Princeton, NJ 08543, USA
}

1. Introduction. The ability of a device to provide adiabatic motion of charged particles is crucial for magnetic confinement. As the magnetic field in the present-day spherical tori, e.g., MAST and NSTX, is much lower than in the conventional tokamaks, effects of the finite Larmor radius (FLR) on the motion of fast ions are of importance in these devices, affecting the stochasticity threshold for the interaction of the ions with electromagnetic perturbations. In addition, FLR by itself may result in nonconservation (jumps) of the magnetic moment of particles [4]. In this work we propose a Lagrangian approach to description of the resonant collisionless motion of charged particles under a perturbation, allowing for FLR. The work generalizes results of Ref. [1], where only time-independent perturbations were considered. The approach is used to find the stochasticity thresholds for the Goldston-White-Boozer (GWB) diffusion [2] and the cyclotron-resonance-induced (CRI) diffusion (for the case of the first cyclotron resonance, the latter was discovered in Ref. [3]). In addition, a new expression for the magnetic moment variation caused by FLR is found.

2. Perturbative approach. We present the exact Lagrangian of the particle motion, $\mathcal{L}$, as the sum of a Lagrangian of an integrable system and the residual considered as a perturbation. As the integrable system, we take the lowest-order Lagrangian of the guiding-center motion [5] in the axisymmetric magnetic field $\mathbf{B}$,

$$
\mathcal{L}_{g c}(\mathbf{X}, \mathcal{E}, \mu, \dot{\mathbf{X}}, \dot{\Phi})=\frac{e}{c} \mathbf{A}(\mathbf{X}) \cdot \dot{\mathbf{X}}+\frac{M v_{\|}}{B} \mathbf{B}(\mathbf{X}) \cdot \dot{\mathbf{X}}+\frac{M c}{e} \mu \dot{\Phi}-\mathcal{E}
$$

where $\mathbf{X}$ is the guiding center position; $e, M, \mu=M v_{\perp}^{2} /[2 \mathbf{B}(\mathbf{X})]$, and $\mathcal{E}$ are the charge, mass, magnetic moment, and energy of the particle; $\Phi$ is the gyrophase; $\mathbf{A}$ is the vector potential; $v_{\|}=\{2[\mathcal{E}-\mu B(\mathbf{X})] / M\}^{1 / 2}$. The guiding center position, $\mathbf{X}$, and the particle position, $\mathbf{x}$, are connected to the considered order by the relation $\mathbf{x}=\mathbf{X}+\rho_{\perp} \hat{\mathbf{a}}$, where $\rho_{\perp}=v_{\perp} / \omega_{B}, \omega_{B}=e B /(M c), \hat{\mathbf{a}}=\cos (\Phi) \hat{\mathbf{e}}_{1}(\mathbf{X})-\sin (\Phi) \hat{\mathbf{e}}_{2}(\mathbf{X}),-\hat{\mathbf{e}}_{1}$ and $\hat{\mathbf{e}}_{2}$ are the binormal and the normal to the magnetic field line, respectively. The Lagrangian given by Eq. (1) indeed describes an integrable system, possessing three constants of motion: $\mathcal{E}$, the canonical angular momemtum $\left(J_{3}\right)$, and $\mu$. Then we present $\mathcal{L}$ as follows:

$$
\mathcal{L}=\mathcal{L}_{g c}+\underbrace{\left(\mathcal{L}_{a s}-\mathcal{L}_{g c}\right)}_{\delta \mathcal{L}_{1}}+\underbrace{\left(\mathcal{L}-\mathcal{L}_{a s}\right)}_{\delta \mathcal{L}_{2}}=\mathcal{L}_{g c}+\delta \mathcal{L}_{1}+\delta \mathcal{L}_{2},
$$

where 


$$
\begin{gathered}
\mathcal{L}(\mathbf{x}, \mathbf{v}, \dot{\mathbf{x}})=\frac{e}{c}[\mathbf{A}(\mathbf{x})+\tilde{\mathbf{A}}(\mathbf{x})] \cdot \dot{\mathbf{x}}+M \mathbf{v} \cdot \dot{\mathbf{x}}-\frac{M}{2} v^{2}-e \tilde{\phi} \\
\mathcal{L}_{a s}(\mathbf{x}, \mathbf{v})=\frac{e}{c} \mathbf{A}(\mathbf{x}) \cdot \dot{\mathbf{x}}+M \mathbf{v} \cdot \dot{\mathbf{x}}-\frac{M}{2} v^{2}
\end{gathered}
$$

is the Lagrangian of the particle motion in the axisymmetric field; $\mathbf{v}$ is the particle velocity; $\phi$ is the scalar potential of the electromagnetic field; tildes refer to the electromagnetic perturbation. We will consider both $\delta \mathcal{L}_{1}$ and $\delta \mathcal{L}_{2}$ as perturbations, the former being responsible for all nonadiabatic effects in the axisymmetric field, and the latter describing the effect of the electromagnetic perturbation.

3. Nonconservation of the magnetic moment. To derive an equation for $\dot{\mu}$ in the axisymmetric field, we set $\delta \mathcal{L}_{2}=0$ and obtain from the Euler-Lagrange equations $[1]$ :

$$
\dot{\mu}=\frac{e}{M c}\left[\frac{\partial \delta \mathcal{L}_{1}}{\partial \Phi}-\frac{d}{d t}\left(\frac{\partial \delta \mathcal{L}_{1}}{\partial \dot{\Phi}}\right)\right]=M \frac{v_{\|}^{2} v_{\perp}}{B R_{c}} \cos \Phi
$$

where $R_{c}$ is the curvature radius of the magnetic field line, and we have omitted terms $\propto \exp (2 i \Phi)$. To compare Eq. (5) with previous results, one should have in mind that here $\mu$ is defined in terms of the magnetic field at the guiding center. Taking the magnetic field at the particle position, as in most previous works, we find:

$$
\frac{d}{d t}\left(\frac{M v_{\perp}^{2}}{2 B(\mathbf{x})}\right)=\dot{\mu}+M \frac{v_{\perp}^{3}}{2 B R_{c}} \cos \Phi-M \frac{2 \pi v_{\perp}^{3}}{B^{3}}\left[\left(\hat{\mathbf{e}}_{2} \cdot \nabla p\right) \cos \Phi+\left(\hat{\mathbf{e}}_{1} \cdot \nabla p\right) \sin \Phi\right] .
$$

The first two terms of Eq. (6) yield the equation for $\delta \mu$ of Refs. [6,7]. Thus, Eq. (5) differs from the mentioned previous formulae by allowing for effects of plasma pressure. Introducing a curvature-like parameter $8 \pi B^{-2} \nabla p$, we could obtain a formula for $\dot{\mu}$ similar to that derived in Ref. [8]. However, the equation for $\dot{\mu}$ given by Eq. (5) is much simpler, while correctly describing the mentioned effects.

4. Lagrangian description of resonant motion. In the action-angle variables the Lagrangian $\mathcal{L}_{g c}$ takes the form

$$
\mathcal{L}_{g c}=J_{1} \dot{\varsigma_{1}}+J_{2} \dot{\varsigma_{2}}+J_{3} \dot{\varsigma_{3}}-\mathcal{E}
$$

where $J_{1}=\mu M c / e ; J_{3}$ is the canonical angular momentum;

$$
J_{2}\left(J_{1}, J_{3}, \mathcal{E}\right)=\frac{1}{2 \pi} \oint d \mathbf{X} \cdot\left[\frac{e}{c} \mathbf{A}(\mathbf{X})+\frac{M v_{\|}}{B} \mathbf{B}(\mathbf{X})\right]
$$

$\varsigma_{i}, i=1,2,3$, are the angle variables canonically conjugate to $J_{i}$ and characterizing the gyrophase, the bounce phase, and the toroidal angle, respectively; the integral in Eq. (8) is taken along a poloidal contour that lies on an invariant torus of the system.

Considering the motion near the resonance $l \omega_{1}-s \omega_{2}-N \omega_{3}+\Omega=0$, where $\omega_{i}=\dot{\zeta}_{i}$, $\Omega$ is the perturbation frequency, we divide the phase-space variables in the vicinity of 
the resonance into "slow" variables $\left(J_{1}, J_{2}, J_{3}\right.$, and $\left.l_{\varsigma_{1}}-s \varsigma_{2}-N_{\varsigma_{3}}+\Omega t\right)$ and "fast" ones (two other independent angular coordinates) and average the Lagrangian over the fast variables. Hence, we introduce the new action-angle variables $\left(I_{1}, I_{2}, I_{3} ; \alpha_{1}, \alpha_{2}, \alpha_{3}\right)$ with $\alpha_{1}=l_{\varsigma_{1}}-s \varsigma_{2}-N \varsigma_{3}+\Omega t, \alpha_{2}=\varsigma_{2}, \alpha_{3}=\varsigma_{1}$. Applying the averaging procedure similar to that described in Ref. [1] to the exact Lagrangian, we obtain

$$
\mathcal{L}=\bar{I}_{1} \dot{\bar{\alpha}}_{1}+I\left(\bar{\alpha}_{1}, \bar{I}_{1}, \bar{I}_{3}, \mathcal{E}\right) \dot{\bar{\alpha}}_{2}+\bar{I}_{3} \dot{\bar{\alpha}}_{3}-H
$$

where bars refer to the new variables modified by the averaging;

$$
I\left(\bar{\alpha}_{1}, \bar{I}_{1}, \bar{I}_{3}, \mathcal{E}\right)=I_{2}\left(\bar{I}_{1}, \bar{I}_{3}, \mathcal{E}\right)+\tilde{I}\left(\bar{\alpha}_{1}, \bar{I}_{1}, \bar{I}_{3}, \mathcal{E}\right)
$$

is the adiabatic invariant of the resonant motion; $I_{2}=J_{2}-(s / N) J_{3} ; H=\mathcal{E}-J_{3} / N$;

$$
\tilde{I}=\frac{\Omega}{8 \pi^{3} N^{3}} \int_{-\pi n_{3}}^{\pi n_{3}} d \varsigma_{10} \int_{-\pi N / \Omega}^{\pi N / \Omega} d t_{0} \int_{L\left(\varsigma_{10}, t_{0}, \alpha_{1}, P, \mu, \mathcal{E}\right)}\left[d \mathbf{x} \cdot \frac{e}{c} \tilde{\mathbf{A}}(\mathbf{x})-d t e \tilde{\phi}\right]
$$

$L\left(\varsigma_{10}, t_{0} \alpha_{1}, J_{3}, \mu, \mathcal{E}\right)$ is the curve in the phase space determined by the equations $-\pi N \leq$ $\varsigma_{2} \leq \pi N, t=t_{0}+\varsigma_{2} / \omega_{2}, \varsigma_{1}=\varsigma_{10}+\left(\omega_{1} / \omega_{2}\right) \varsigma_{2}, \varsigma_{3}=\left(l_{\varsigma_{1}}-s \varsigma_{2}+\Omega t-\alpha_{1}\right) / N, J_{3}=$ const, $\mu=$ const, $\mathcal{E}=$ const. The adiabatic invariant $I$ can be used to determine the width of resonance islands and evaluate the threshold amplitude of the perturbation at which the transition to global stochasticity takes place due to the overlap of the islands.

5. Stochasticity threshold for the motion in a rippled magnetic field. When the perturbation in Eq. (11) is the magnetic field ripple, the integrand is typically a rapidly oscillating function. It is known that the main contribution to such integrals comes from the vicinities of the points of stationary phase, which at the exact global resonance coincide with the points of "local" resonance, $l \omega_{B}=N \dot{\varphi}$, where $N$ is the number of the toroidal field coils, $\varphi$ is the toroidal angle (one should distinguish the local resonance from the "global" one, $l \omega_{1}-s \omega_{2}-N \omega_{3}+\Omega=0$ ). Typically, there are two points of the local resonance for a given $l$ on a particle orbit. Then, in the simplest case of up-down symmetry, we obtain [1]:

$$
I=\left.\frac{N}{2 \pi} \varphi_{p}^{\prime}\right|_{I_{1}=I_{1 r}}\left(I_{1}-I_{1 r}\right)^{2}+\frac{1}{\pi} \delta I_{1}\left(I_{1}, I_{3}, \mathcal{E}\right) \cos \left[\frac{N}{2} \varphi_{b}\left(I_{1}, I_{3}, \mathcal{E}\right)\right] \cos \alpha_{1},
$$

where " $r$ " refers to the value at the global resonance, prime denotes $\partial / \partial I_{1}=-N \partial / \partial J_{3}+$ $(l e / M c) \partial / \partial \mu, \delta I_{1}$ characterizes the jump of $I_{1}$ for one local resonance event, $\varphi_{p}$ and $\varphi_{b}$, the unidirectional and alternate parts of the perturbation phase variation between the events. We observe that the mathematical description of the resonant motion for different $l$ is rather similar. The transition to chaos for the GWB diffusion $(l=0)$ was studied in Ref. [9]. The transition occurs in two different ways, depending on the parameter $Q=\phi_{b}^{\prime}\left(N \delta I_{1} / \phi_{p}^{\prime}\right)^{1 / 2}$. When $Q$ is small, the level contours of $I$ describe a conventional Chirikov island with the width $\propto \delta I_{1}^{1 / 2}$. When $Q$ exceeds $\sim 2$, the contours show chains of islands near the resonance, the width of the chain being $\propto \delta I_{1}$. 
Using the mentioned similarity, we generalize the known results for $l=0$ to arbitrary $l$ and obtain the following stochasticity criterion for the field ripple of the form $\delta_{\text {rip }}=$ $\delta_{0} \exp \left(R / L_{\text {rip }}\right)$, where $R$ is the distance to the axis of symmetry, $\delta_{0}$ is a constant:

$$
\bar{\delta}_{r i p}>\delta_{c r i t} \equiv \frac{(2|\Xi|)^{1 / 2} c}{(\pi N)^{1 / 2} e \bar{B} v_{\perp} L_{r i p} a_{l}\left(\rho_{\perp} / L_{\text {rip }}\right)\left(\left|\varphi_{p}^{\prime}\right|+\left|\varphi_{b}^{\prime}\right|\right)},
$$

where all quantities are taken at the local resonance point, bars refer to the guiding center, $\left.a_{l}(x)=\left[\mathrm{I}_{|l-1|}\right)(x)+\mathrm{I}_{|l+1|}(x)\right] / 2, \mathrm{I}_{j}(x)$ are modified Bessel functions,

$$
\Xi=\frac{v^{2}}{2 q R^{2}} \frac{\partial \ln B}{\partial \theta}\left(1+\frac{l^{2} R^{2}}{N^{2} \rho^{2}}\right)+\frac{l \omega_{B}}{N} \frac{d \ln R}{d t} .
$$

$\theta$ is the poloidal angle. This criterion agrees with previously obtained criteria for the GWB diffusion $(l=0)$ for $\rho \ll L_{\text {rip }}$ but allows for the effect of FLR. For instance, this effect results in a decrease of $\delta_{\text {crit }}$ by a factor of 1.5 for $\rho_{\perp} / L_{r i p}=2$, as is the case for $80-\mathrm{keV}$ deuterons in NSTX. Equation (13) may not agree with previously obtained stochasticity thresholds for the CRI diffusion, especially for large magnitudes of $N \rho / R$, because the previous results were obtained without taking account of jumps in $J_{3}$.

6. Summary. A Lagrangian formalism for the description of the nonadiabatic effects on the particle motion is developed. Based on this formalism, a general expression for the critical ripple amplitude is obtained, which for the GWB diffusion of particles with $\rho \ll L_{\text {rip }}$ agrees with previous results. In addition, a formula for the variation of the magnetic moment due to FLR is derived. The formula agrees with that obtained by a different procedure in Refs. [6,7] but takes into account effects of the plasma pressure and has a simpler form.

Acknowledgments. The work was supported by the Awards No. UP2-2114 and UP2-2419-KV-02 of CRDF and the Government of Ukraine, the U.S. DoE under contract number DE-AC02-76-CHO3073, and the Research Contract No. 10539 of the IAEA. The presenting author (Y.Y.) acknowledges the support of the Local Organizing Committee for his trip to the Conference.

\section{REFERENCES}

[1] Ya. I. Kolesnichenko, R. B. White, Yu. V. Yakovenko, Phys. Plasmas 9, 2639 (2002).

[2] R. J. Goldston, R. B. White, A. H. Boozer, Phys. Rev. Lett. 47, 647 (1981).

[3] S. V. Putvinskiǔ, JETP Lett. 36, 397 (1982).

[4] A. Garren et al., in Proc. 2nd UN Int. Conf. on the Peaceful Uses of Atomic Energy, Geneva, 1958 (UN, Geneva, 1958), Vol. 31, p. 65.

[5] R. G. Littlejohn, J. Plasma Phys. 29, 111 (1983).

[6] R. J. Hastie, G. D. Hobbs, J. B. Taylor, in Plasma Physics and Controlled Nuclear Fusion Research, Conf. Proc., Novosibirsk, 1968 (IAEA, Vienna, 1969), Vol. 1, p. 389.

[7] J. E. Howard, Phys. Fluids 14, 2378 (1971).

[8] R. H. Cohen, G. Rowlands, J. H. Foote, Phys. Fluids 21, 627 (1978).

[9] R. B. White, Phys. Rev. E 58, 1774 (1998). 


\section{External Distribution}

Plasma Research Laboratory, Australian National University, Australia

Professor I.R. J ones, Flinders University, Australia

Professor J oão Canalle, Instituto de Fisica DEQ/IF - UERJ , Brazil

Mr. Gerson O. Ludwig, Instituto Nacional de Pesquisas, Brazil

Dr. P.H. Sakanaka, Instituto Fisica, Brazil

The Librarian, Culham Laboratory, England

Library, R61, Rutherford Appleton Laboratory, England

Mrs. S.A. Hutchinson, JET Library, England

Professor M.N. Bussac, Ecole Polytechnique, France

Librarian, Max-Planck-Institut für Plasmaphysik, Germany

J olan Moldvai, Reports Library, MTA KFKI-ATKI, Hungary

Dr. P. Kaw, Institute for Plasma Research, India

Ms. P.J . Pathak, Librarian, Insitute for Plasma Research, India

Ms. Clelia De Palo, Associazione EURATOM-ENEA, I taly

Dr. G. Grosso, Instituto di Fisica del Plasma, Italy

Librarian, Naka Fusion Research Establishment, J AERI, J apan

Library, Plasma Physics Laboratory, Kyoto University, J apan

Research Information Center, National Institute for Fusion Science, J apan

Dr. O. Mitarai, Kyushu Tokai University, J apan

Library, Academia Sinica, Institute of Plasma Physics, People's Republic of China

Shih-Tung Tsai, Institute of Physics, Chinese Academy of Sciences, People's Republic of China

Dr. S. Mirnov, TRINITI, Troitsk, Russian Federation, Russia

Dr. V.S. Strelkov, Kurchatov Institute, Russian Federation, Russia

Professor Peter Lukac, Katedra Fyziky Plazmy MFF UK, Mlynska dolina F-2, Komenskeho Univerzita, SK-842 15 Bratislava, Slovakia

Dr. G.S. Lee, Korea Basic Science Institute, South Korea

Mr. Dennis Bruggink, Fusion Library, University of Wisconsin, USA

Institute for Plasma Research, University of Maryland, USA

Librarian, Fusion Energy Division, Oak Ridge National Laboratory, USA

Librarian, Institute of Fusion Studies, University of Texas, USA

Librarian, Magnetic Fusion Program, Lawrence Livermore National Laboratory, USA

Library, General Atomics, USA

Plasma Physics Group, Fusion Energy Research Program, University of California at San Diego, USA

Plasma Physics Library, Columbia University, USA

Alkesh Punjabi, Center for Fusion Research and Training, Hampton University, USA

Dr. W.M. Stacey, Fusion Research Center, Georgia Institute of Technology, USA

Dr. J ohn Willis, U.S. Department of Energy, Office of Fusion Energy Sciences, USA

Mr. Paul H. Wright, Indianapolis, Indiana, USA 
The Princeton Plasma Physics Laboratory is operated by Princeton University under contract with the U.S. Department of Energy.

\author{
Information Services \\ Princeton Plasma Physics Laboratory \\ P.O. Box 451 \\ Princeton, NJ 08543
}

Phone: 609-243-2750

Fax: 609-243-2751

e-mail: pppl_info@pppl.gov

Internet Address: http://www.pppl.gov 